\title{
$\underline{\mathbf{P}-148}$
}

\section{Scopoletin from the Flowers of Malaysian Uncaria Cordata}

\author{
Nur Hakimah Abdullah and Rohaya Ahmad* \\ Faculty of Applied Sciences, Universiti Teknologi MARA, 40450, Shah Alam, Selangor; \\ E-mail: rohayaahmad@salam.uitm.edu.my
}

We have reported the isolation of pentacyclic oxindole alkaloids from Uncaria longiflora (Salim et al., 2011). Continuing our interest in the genus, we have selected Uncaria cordata which was collected during the flowering season for phytochemical investigation. The crude methanolic extract of the flowers was subjected to column chromatography using normal phase silica employing DCM:EtOAc followed by EtOAc:MeOH as the solvent system to give seven fractions. Repeated preparative thin layer chromatography on Fraction 2 successfully yielded scopoletin (7-hydroxy-6-methoxy coumarin). The structure of the compound was elucidated on the basis of extensive spectroscopic data analysis, mostly 1D-NMR, 2D-NMR as well as comparison with literature. HPLC profiling of the methanolic extracts of the flowers, hooks, roots and leaves on Agilent 1200 system (DAD) using an RP-C18 column showed the presence of this compound in the different parts of this plant along with other secondary metabolites.

Keyword: Uncaria cordata, Scopoletin, Coumarin. 